\title{
HIGH-POWER TESTING OF 11.424-GHz DIELECTRIC-LOADED ACCELERATING STRUCTURES*
}

\author{
S.H. Gold, Plasma Physics Division, NRL, Washington, DC 20375, USA \\ W. Gai, High Energy Physics Division, ANL, Argonne, IL 60439, USA
}

\section{Abstract}

The design, construction, and bench testing of an $\mathrm{X}$ band travelling-wave accelerating structure loaded with a permittivity $=20$ dielectric has been published recently by the Argonne Advanced Accelerator Group [1]. Here we describe a new program to build a test accelerator using this structure. The accelerator will be powered using high-power 11.424-GHz radiation available at the Magnicon Facility at the Naval Research Lab [2]. The magnicon is expected to provide up to $30 \mathrm{MW}$ from each of two WR-90 output waveguide arms in pulses of up to 1- $\mu$ s duration, permitting tests of the dielectric-loaded X-band device at gradients of $\sim 40 \mathrm{MV} / \mathrm{m}$. The use of higher power pulses (100-500 MW) eventually available at the output of an active pulse compressor [3] driven by the magnicon will permit gradients in excess of $100 \mathrm{MV} / \mathrm{m}$ to be achieved.

\section{INTRODUCTION}

The investigation of new types of high performance accelerating structures has been a major area of research in recent years [4]. Proposals to use rf-driven dielectric-loaded waveguides as particle accelerators date from the 1950s [5]; however, to date, there has been no experimental investigation of these structures at high power. The potential advantages of using dielectric accelerating structures are: simplicity of construction; comparable shunt impedance to conventional copper accelerating structures; simplicity of implementing deflection-mode damping; and the fact that the maximum electric field amplitude anywhere in the structure is the axial accelerating field.[6]. There are also challenges for dielectric-loaded structure technology because of the potential of dielectric breakdown at high power and rf-induced joule heating of the loading medium. These issues can only be settled by high power, high gradient testing of the device. In this paper, we discuss the efforts of a collaboration between NRL and ANL on high power tests of both traveling- and standingwave X-band dielectric loaded devices using the NRL Magnicon Facility [2], with the goal of obtaining 50-100 $\mathrm{MV} / \mathrm{m}$ acceleration gradients in these structures.

\section{PROPERTIES OF DIELECTRIC- LOADED ACCELERATOR}

\subsection{Traveling-wave (TW) Accelerator}

Figure 1 shows a schematic diagram of a dielectric-

\footnotetext{
*Work supported by the High Energy Physics Division, Advanced Technology Branch, US Department of Energy and the Office of Naval Research.
}

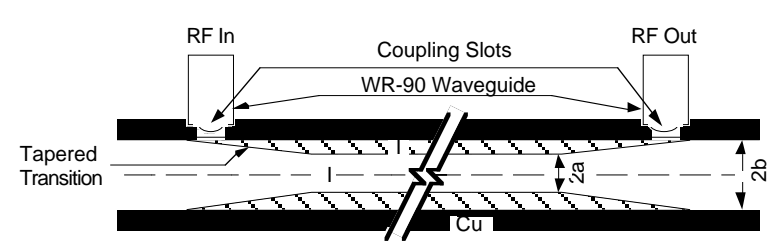

Figure 1. Schematic diagram of the dielectric-loaded traveling-wave accelerating structure.

loaded accelerating structure. One of the difficult engineering challenges in this device is the achievement of efficient coupling from the external rf source to the structure because the outer diameter of the dielectric is much smaller than the rectangular X-band coupling waveguide. We found that by using a combination of side-coupled slots and tapering the inner diameter of the dielectric near the coupling slots, one can efficiently couple the rf from the rectangular waveguide to the dielectric waveguide. The prototype TW dielectric accelerating structure is shown in Fig. 2. In the prototype $11.4 \mathrm{GHz}$ dielectric-loaded accelerator, we have achieved $>95 \%$ power coupling. The parameters of the structure are given in Table 1.

\subsection{Standing-wave (SW) Accelerator}

We have also constructed a standing-wave accelerator using the same class of ceramic but with a slightly different dielectric constant $(\varepsilon=17)$. The optimal coupling $(-30 \mathrm{~dB})$ was achieved by adjusting the coupling slot size and taper position. The measured Q is 2200 , which agrees well with the predicted value of 2400 . The expected

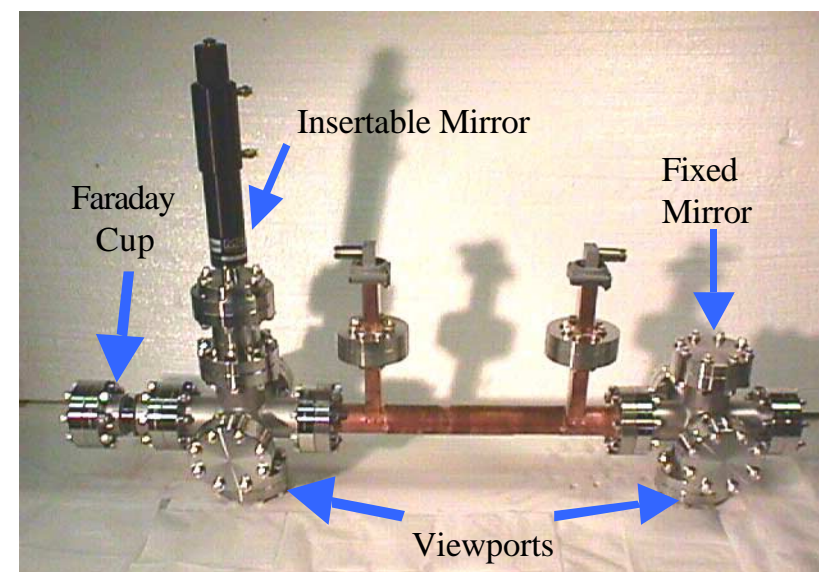

Figure 2. Assembled traveling-wave dielectric accelerator for the NRL high power test. The mirrors will be used for monitoring arcing from dielectric surface breakdown. Forward and reflected rf power will be measured, and a Faraday cup will collect and measure dark current. 
Table 1. TW structure specifications

\begin{tabular}{|l|l|}
\hline Material & $\mathrm{MgCaTi}$ \\
\hline Permittivity $\varepsilon$ & 20 \\
\hline Taper Angle & $8^{\circ}$ \\
\hline Loss Tangent $\delta$ & $10^{-4}$ \\
\hline Inner Radius a & $0.3 \mathrm{~cm}$ \\
\hline Outer Radius b & $0.456 \mathrm{~cm}$ \\
\hline Group Velocity & $0.057 \mathrm{c}$ \\
\hline Attenuation & $4 \mathrm{~dB} / \mathrm{m}$ \\
\hline Power for $10 \mathrm{MV} / \mathrm{m}$ & $2.6 \mathrm{MW}$ \\
\hline
\end{tabular}

acceleration gradient is $25 \mathrm{MV} / \mathrm{m}$ for 6-MW input $\mathrm{rf}$ power. We are currently designing the cooling, vacuum, and mechanical fixtures for this structure; this SW accelerator will be ready for its high power test shortly after the TW structure tests are completed. Figure 3 and 4 shows the structure under construction and the network analyzer measurement of $S_{11}$ showing the resonant frequency and quality factor.

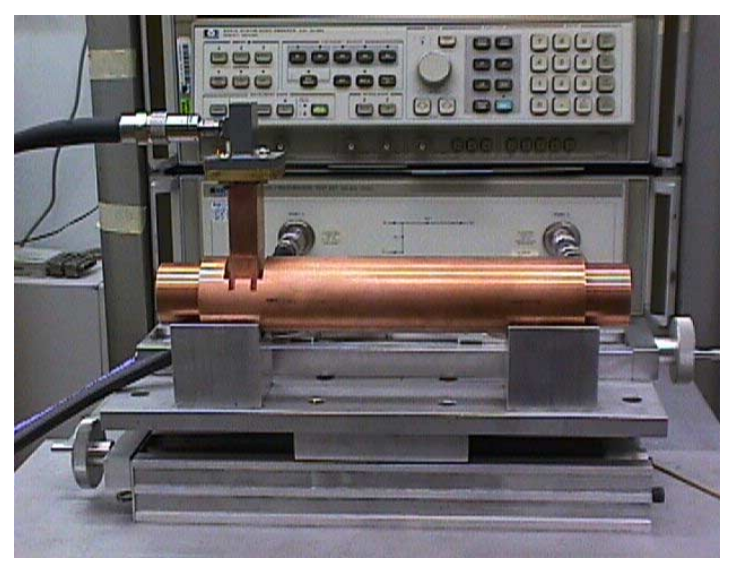

Figure 3. X-band SW accelerator structure in tuning configuration.

\section{NRL MAGNICON FACILITY}

The magnicon (see Fig. 5) is a "scanning-beam," or deflection-modulated amplifier tube that offers the potential for high power and very high efficiency at frequencies ranging from $1 \mathrm{GHz}$ to $35 \mathrm{GHz}$. The $11.424-\mathrm{GHz}$ magnicon development program [2] has been carried out as a collaboration between NRL and Omega-P, Inc., with the goal of developing a competitor to the SLAC X-band klystron as a source for powering future colliders. It is designed to produce $\sim 60 \mathrm{MW}$ of power, equally divided between two output arms, with an adjustable pulse length of up to $1.2 \mu$ s, and a repetition rate of up to $10 \mathrm{~Hz}$. It is engineered like a conventional high-power klystron, with OFHC copper and stainless steel construction and hightemperature bakeout. Electric fields in the electron gun and surface rf-electric fields in the cavities are below well-

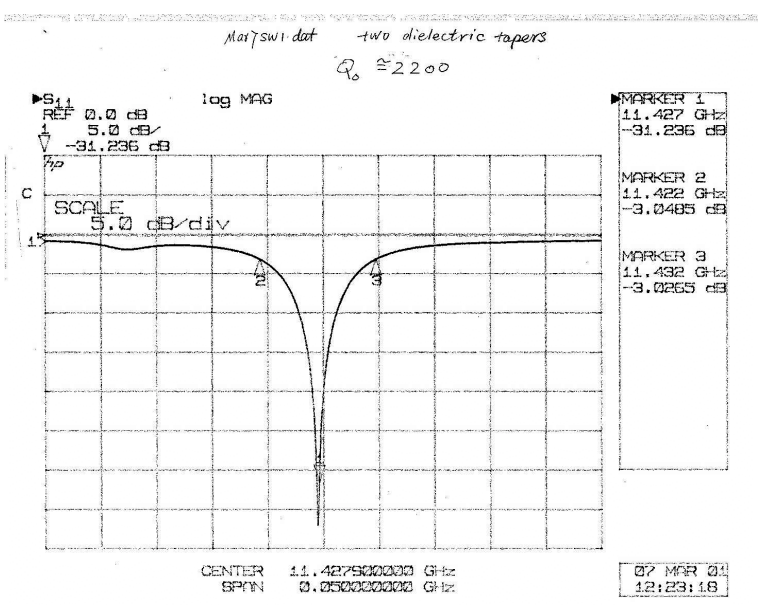

Figure 4. Tuning curve ( $\mathrm{S}_{11}$ vs frequency) of the SW structure using the HP network analyzer.

established breakdown limits. The base pressure of the gun and rf circuit is $\sim 1 \times 10^{-9} \mathrm{~T}$. The output is in two conventional WR-90 waveguides, and all output components, including flanges, windows, pumping tees, directional couplers, and loads, are of SLAC design. Its frequencystable drive signal is provided by a microwave sweep oscillator monitored by a frequency counter, and amplified by a $1-\mathrm{kW}$ traveling-wave tube (TWT) amplifier. Rf conditioning is still in progress. However, $25 \mathrm{MW}$ (12.5 MW/arm) has already been produced in a 200-ns output pulse, permitting TW tests at up to $20 \mathrm{MV} / \mathrm{m}$ and $\mathrm{SW}$ tests at up to $40 \mathrm{MV} / \mathrm{m}$. The magnicon is also relatively insensitive to mismatched or reactive loads, making it an ideal source for testing accelerating structures and pulsecompressor cavities. Following the completion of the conditioning, it will be used primarily as a high-power source to test advanced accelerator and active pulse compressor concepts.

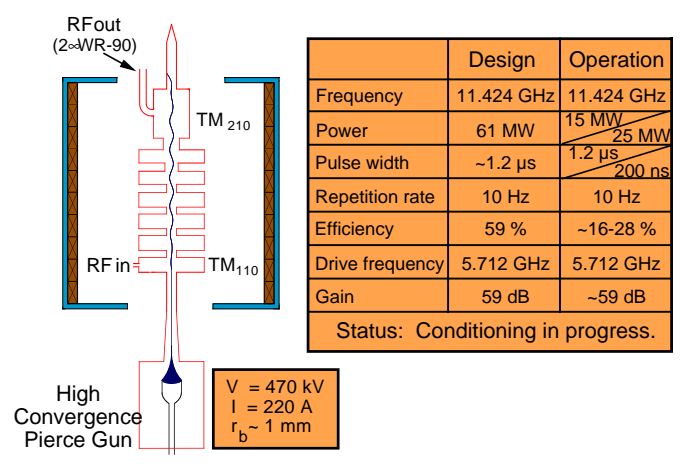

Figure 5. Magnicon schematic and parameters

The NRL magnicon facility (see Fig. 6) occupies a $\sim 110-\mathrm{m}^{2}$ high-bay vault, with a $6-\mathrm{m}$ ceiling height and a 5 -ton bridge crane, with 2.5 -m-thick concrete walls and ceiling, and shielded entryways. Within the vault, the magnicon is situated behind a $2.5-\mathrm{m}$ high, $1 / 2$-m-thick 
concrete shielding wall. A $6.5 \times 1.5-\mathrm{m}$ pulse-compressor platform, 2.5-m above the level of the floor, is located adjacent to the magnicon, and extends over the shielding wall. Lead shielding is employed around the electronbeam collector of the magnicon tube to minimize "skyshine," and will also be employed around the beam dump of low-current (i.e., dark-current) dielectric-loaded accelerator experiments. However, for the first test of the accelerator with an injected beam, we plan to build a concrete bunker with 1-m walls and ceiling within the vault. This should provide sufficient shielding for low-average-current experiments at up to $20-\mathrm{MeV}$ final electron energy.

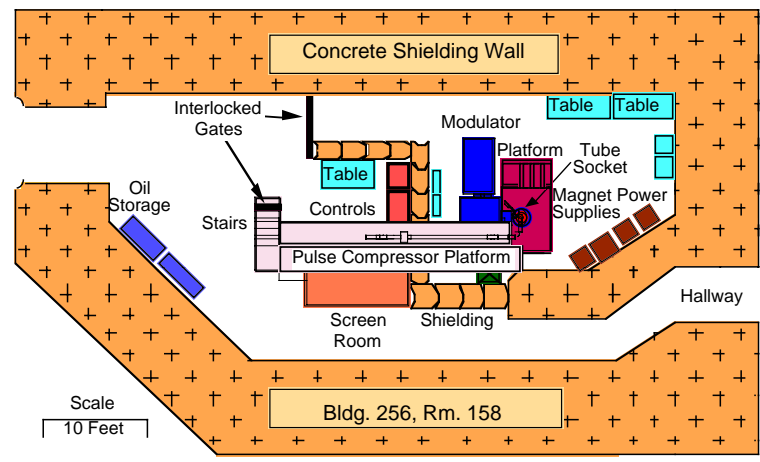

Figure 6. NRL Magnicon Facility

\section{HIGH POWER TEST EXPERIMENT AND ELECTRON BEAM ACCELERATION}

Given the capabilities of the NRL magnicon of source described above, we have defined a test plan for the TW and SW structures:

1. Dielectric breakdown test: The most relevant question we need to answer about dielectric-loaded structures is the onset of breakdown under high rf fields. Early experiments suggest that these dielectrics can easily sustain $>10 \mathrm{MV} / \mathrm{m}$ gradient. In this phase of the experiment, we should be able to test at up to 40 $\mathrm{MV} / \mathrm{m}$ gradients for TW using the combined rf output from both arms. For the same rf power of $50 \mathrm{MW}$, we can test the SW structure to $80 \mathrm{MV} / \mathrm{m}$. If these structures can sustain fields at this level, then we can classify them as high gradient structures. With the higher rf power levels (100-500 MW) expected to be available from the ongoing NRL pulse compression experiment, one could test both TW and SW structures at $>100 \mathrm{MV} / \mathrm{m}$ gradients. These experiments will also be sensitive to any field or radiation damage induced changes in the dielectric properties of the medium through increases in reflected power from shifts in the resonant frequency of the device.

2. Electron beam acceleration: Upon successful demonstration of high gradients in dielectric structures, we will construct and install a 4-MeV compact X-band injector [7] in order to conduct electron beam acceleration experiments. Using the current $25-\mathrm{cm}$-long TW structure, $10 \mathrm{MeV}$ net acceleration should be achievable. During this stage of the experiment, the energy of the injected electron beam will be measured with a magnetic dipole spectrometer.

3. Demonstration of a compact dielectric accelerator with acceleration length of $50 \mathrm{~cm}$, thus obtaining 20-40 $\mathrm{MeV}$ energy gains. This would require a higher precision tuning and machining of the dielectric tubes used to fabricate the structures. We do not anticipate that this will provide any unusual difficulties.

\section{SUMMARY}

We have devised a detailed plan for high power tests of dielectric-loaded accelerating structures at NRL using the NRL Magnicon Facility. Through these experiments, we should be able to answer remaining questions of the feasibility of the dielectric-based concept, particularly emphasizing issues related to high-gradient breakdown. We plan to construct and test a compact accelerator if the high power tests are successful.

\section{REFERENCES}

[1] P. Zou et al., Rev. Sci. Instrum. 71, 2301, 2000.

[2] O.A. Nezhevenko et al., paper at this conference.

[3] A.L. Vikharev et al., Proc. $9^{\text {th }}$ Workshop on Advanced Accelerator Concepts, in press.

[4] J. Rosenzweig, "Summary report: Working Group 3 on 'Structure-based acceleration'," in Advanced Accelerator Concepts, AIP Conference Proceedings 398, edited by S. Chattopadhyay, J. McCullough, and P. Dahl (AIP, New York, 1997), pp. 181-186.

[5] G.T. Flesher and G.I. Cohn, AIEE Trans. 70, 887, 1951.

[6] P. Zou, W. Gai, R. Konecny, X. Sun, and T. Wong, "X-Band Dielectric Loaded Traveling-wave Acceleration Structure," in Proceedings of the Ninth Workshop on Advanced Accelerator Concepts, Santa Fe, NM, 2000, in press.

[7] X. Sun, "6 MeV X-band on-axis standing wave linear accelerator," ANL Note WF-200. 